\title{
Addendum to the paper: Hyperelasticity as a $\Gamma$-limit of peridynamics when the horizon goes to zero
}

\author{
José C. Bellido $^{1}$ - Carlos Mora-Corral ${ }^{2}$. \\ Pablo Pedregal ${ }^{1}$
}

Received: 2 March 2015 / Published online: 26 March 2015

(C) Springer-Verlag Berlin Heidelberg 2015

In the above article [1] the funding information was missing. Please find it below:

Acknowledgments This work has been supported by the Spanish Ministerio de Economía y Competitividad through grants MTM2013-47053-P (Bellido and Pedregal) and MTM2011-28198 (Mora-Corral), the Consejería de Educación, Cultura y Deportes of the Spanish Junta de Comunidades de Castilla-La Mancha and the European Fund for Regional Development through grant PEII-2014-010-P (Bellido and Pedregal), the ERC Starting Grant 307179 (Mora-Corral), and the European Fund and the Spanish Ramón y Cajal programme through grant RYC-2010-06125 (Mora-Corral).

\section{Reference}

1. Bellido, J.C., Mora-Corral, C., Pedregal, P.: Hyperleasticity as a $\Gamma$-limit of peridynamics when the horizon goes to zero. Calc. Var. (2015). doi:10.1007/s00526-015-0839-9

Communicated by L. Ambrosio.

Carlos Mora-Corral

carlos.mora@uam.es

José C. Bellido

JoseCarlos.Bellido@uclm.es

Pablo Pedregal

Pablo.Pedregal@uclm.es

1 E.T.S.I. Industriales, Department of Mathematics, University of Castilla-La Mancha, 13071 Ciudad Real, Spain

2 Department of Mathematics, Faculty of Sciences, University Autónoma of Madrid, 28049 Madrid, Spain 